\title{
Epilepsy in the X-linked Syndromic Intellectual Disability, Christianson Type Resulted from c.584+1 G>T SLC9A6 Variant
}

\author{
Justyna Paprocka ${ }^{1 *}$, Agnieszka Charzewska ${ }^{2}$, Dorota Hoffman-Zacharska ${ }^{2}$, Ewa Emich-Widera ${ }^{1}$ \\ and Aleksandra Jezela-Stanek ${ }^{3}$ \\ ${ }^{1}$ Department of Pediatric Neurology, Faculty of Medical Science in Katowice, Medical University of Silesia, Poland \\ ${ }^{2}$ Department of Medical Genetics, Institute of Mother and Child, Warsaw, Poland \\ ${ }^{3}$ Department of Genetics and Clinical Immunology, National Institute of Tuberculosis and Lung Disease, Poland
}

*Corresponding author: Justyna Paprocka, Department of Pediatric Neurology, Faculty of Medical Sciences in Katowice, Medical University of Silesia, ul. Medyków 16, 40-752 Katowice, Poland

To Cite This Article: Justyna P, Agnieszka C, D Hoffman-Zacharska, E Emich-Widera, A Jezela-Stanek. Epilepsy in the X-linked Syndromic Intellectual Disability, Christianson Type Resulted from c.584+1 G>T SLC9A6 Variant. 2020 - 8(3). AJBSR.MS.ID.001278. DOI: 10.34297/ AJBSR.2020.08.001278.

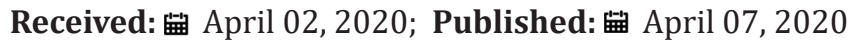

\begin{abstract}
Christianson syndrome (CS) is an X-linked intellectual disorder caused by mutations in the SLC9A6 gene (Xq26 locus, XLD inheritance). It is characterized by specific facial and behavioral features, postnatal microcephaly, absent speech, truncal ataxia and epilepsy. We present a history of a 2, 5-year-old boy diagnosed with refractory epilepsy with polymorphic seizures (tonic-clonic, myoclonic, atypical absence and atonic seizures), diagnosed at the age of 15 months. The causative genetic variant (c.584+1 G>T) has been reported to date only in one family, thus with our paper we aimed to delineate the genotype-phenotype correlation in CS.
\end{abstract}

Keywords: Christianson syndrome; Electroencephalography, Epilepsy, Lennox-Gastaut Syndrome, SLC9A6 gene

\section{Introduction}

Christianson syndrome (CS, MIM300243), also known as Mental Retardation, X-Linked, Syndromic, Christianson Type (MRXSCH), is an X-linked neuro developmental disorder of dominant inheritance, characterized by microcephaly, severe global developmental delay with lack of speech, abnormal movements (truncal ataxia, hyperkinetic movements), and early-onset seizures of variable types [1]. Affected children present specific facial dysmorphism, encompassing long, narrow face with square, prognathic and pointed jaw, deep-set eyes, bushy eyebrows, long, straight nose and open-mouth appearance. Moreover, behavioral manifestations, as autistic features, happy demeanor and easily provoked laughter are typical.

Given the phenotypical similarity to Angel man syndrome (AS, MIM 105830) CS was proposed to be included in differential diagnosis of AS. According to the diagnostic criteria for MRXSCH, described by Pescosolido et al. core and secondary diagnostic symptoms should be considered [2]. The core ones (noted in over $85 \%$ of patients) include early-onset in boys, nonverbal status, moderate to severe intellectual disability, epilepsy, truncal ataxia, postnatal microcephaly and/or attenuation in growth of head circumference, and hyperkinetic behavior. In turn, secondary symptoms (presented in over 35\% of patients) encompass autistic behavior and/or Angel man-like phenotype, eye movement abnormalities, developmental regression, particularly loss of independent ambulation after 10 years of age, low weight for age, and cerebellar vermis atrophy, particularly after 10 years of age.

We present a case of a 2,5-year-old boy with refractory epilepsy with polymorphic seizures (tonic-clonic, myoclonic, atypical absence and atonic seizures), diagnosed at the age of 15 months. 


\section{Case Description}

The child was born from the third pregnancy, at term, by Caesarian section (in the second pregnancy stillbirth due to insufficiency of the umbilical vessels) with a birth weight of $3200 \mathrm{~g}$, head circumference of $33 \mathrm{~cm}$, and 10 points in the Apgar score. In the family history psychomotor delay in patient's sister has been observed. Otherwise, it is unremarkable.

The psychomotor development of the boy was retarded. Despite physiotherapy, the child started to sit unsupported at the age of 10 months, to walk with support at the age of 22 months, now (aged 2, 5 years) he cannot speak. The EEG examination showed almost constant generalized (with the temporal and parie to-occipital predominance) paroxysmal changes with spikes, polyspikes, sharp waves, sharp wave-slow wave complexes, slow spikewave complexes, poly spikes-slow wave complexes, slow waves with maximal amplitude to $620 \mathrm{uV}$. He was diagnosed with LennoxGastaut syndrome. The MRI displayed normal brain structures.

In the neurological examination at the age of 2, 5 years microcephaly $(45 \mathrm{~cm},<3$ percentile), generalized hypotonic with brisk deep tendon reflexes, truncalataxia and stereotyped limbs' movements were observed. In physical examination steadily smiling appearance with dysmorphic features, as long, narrow face, square, prognathic, pointed jaw, open mouth with drooling and large ears were noted. The presented happy demean our and easily provoked laughter.

The child was treated with valproic acid (VPA) first. Then, because of the poor efficacy,phenobarbital (PB), clobazam (CLB) and later levetiracetam (LEV) were added. Now, aged 2,5 years, he has been seizures free for three months (on polytherapy: VPA $28 \mathrm{mg} / \mathrm{kg}$, CLB 0,5mg/kg, LEV $60 \mathrm{mg} / \mathrm{kg}$ ). During different antiepileptic treatment, the EEG tracing was always abnormal with constant epileptiform discharges. The concentration of valproic acid and levetiracetam in blood was within normal limits.

\section{Genetic Result}

In order to identify the genetic basis of these diseases, whole exome sequencing (WES), followed by a targeted analysis, was performed for the patients (the exome was captured using the Sure Select Human All Exons v.6, reagents; sequencing formed on the Illumina HiSeq platform, annotations according to Dept. of Med. Genet IMCh pipeline, mutation confirmation by Sanger sequencing). Analysis was performed for the genes related to epilepsy syndromes according to Genomics England Panel App and OMIM Early Infantile Epileptic Encephalopathy Series PS308350.

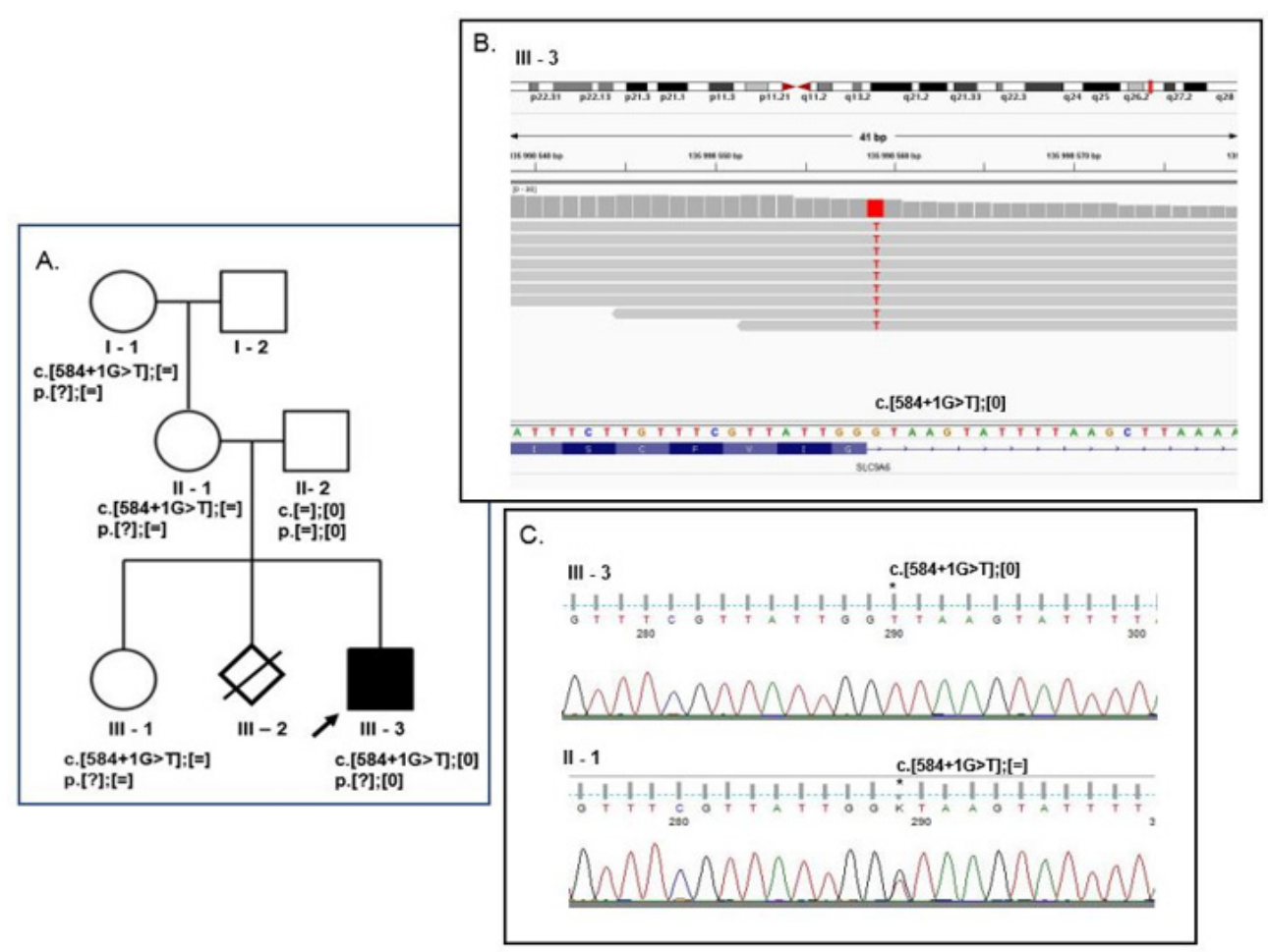

Figure 1: Mutation of the SLC9A6 gene c.584+1G>C , affecting the splicing site, identified in proband with the diagnosis of Intellectual Disability (ID) finally characterized as Christianson type of X-linked syndromic mental retardation (MRXSCH).

a. Pedigree of probands family (III-3 affected proband, III- 1his sister and I-1 and II-1 women who are an asymptomatic carriers of the mutation)

b. NGS data analyzed with IGV

c. Sanger sequencing data analyzed with Finch TV software both showing single nucleotide substitution in proband III-3 (T hemizygote) and his mother I - 2 ( $\mathrm{G} / \mathrm{T}$ heterozygote). 
The performed analysis showed in the SLC9A6 gene the presence of single nucleotide substitution at the invariant GT donor splice site of intron 4-c.584+1 G>T [NM_0063359; GRCh38] (Figure 1). According to insilico analysis it may abolish the natural donor site and/or activate an intronic cryptic donor site, affecting or altering the spilicing. Any errors during the splicing process may lead to improper intron removal and thus cause alterations of the open reading frame. The identified mutation is the known pathogenic variant [HGMD Professional 2020.1; CS133651] described as a background of Mental retardation, X-linked, Christianson type [3]. Further family study revealed proband's sister, mother andgrandmother as an asymptomatic carrier of the mutation (Figure 1).

\section{Discussion}

According to Pescosolido et al. [2], variants in the SLC9A6 gene may be one of the most common causes of X-linked neuro developmental disorders, affecting from 1 in 16,000 to 1 in 100,000 people worldwide [2014]. To date, epilepsy in several cases of Christianson syndrome have been characterized, and recently reviewed by Ikeda et al. [4]. Because of missing epilepsy description, the authors did now however mention and discussedthe paper published in 2013 by Riess et al. who gave first description of two German families with then novel SLC9A6 mutations, the same as identified in presented proband (c.584+1 G>T substitution) [3]. Comparing to the description of the patient gave by Riess the first epileptic seizure in our patient appeared 5 months later. However, these publication dos not mentioned about morphology and treatment epileptic seizures. Hence, our description is the first in which epilepsy in CS is in detail characterized.

The older sister of our patient was found to be a carrier of the same substitution. She suffered from mild intellectual disability, had significant problems with reading, but no seizures were observed. It is unfortunately hard to prove, but, given that female carriers may be mildly affected [5], her clinical status may be a result of this geno type and skewed X-chromosome inactivation.

Referring to the first description of c.584+1 G>T variant. Based on medical history of family 1 (stop codon mutation), Riess and collaborates [3] suggest a link between this variant and late-onset neurodegenerative disorders (parkinsonism).Since our proband's grandmother, being a carrier, did not present any neurological problems, we cannot support the hypothesis suggested by mentioned authors.

\section{Conflict of interest}

The authors declare no conflict of interest

\section{References}

1. Christianson AL, Stevenson RE, van der Meyden CH, Pelser J, Theron FW, et al. (1999) X linked severe mental retardation, craniofacial dysmorphology, epilepsy, ophthalmoplegia, and cerebellar atrophy in a large South African kindred is localised to Xq24-q27. J Med Genet 36: 759-766.

2. Pescosolido MF, Stein DM, Schmidt M, El Achkar CM, Sabbagh M, et al. (2014) Genetic and phenotypic diversity of NHE6 mutations in Christianson syndrome. Ann Neurol 76(4): 581-593.

3. Riess A, Rossier E, Krüger R, Dufke A, Beck-Woedl Set al. (2013) Novel SLC9A6 mutations in two families with Christianson syndrome. Clin Genet 83(6): 596-597.

4. Ikeda A, Yamamoto A, Ichikawa K, Tsuyusaki Y, Tsuji M, Iai M, et al. (2019) Epilepsy in Christianson syndrome: Two cases of Lennox-Gastaut syndrome and a review of literature. Epilepsy Behav Rep 5(13): 100349.

5. Schroer RJ, Holden KR, Tarpey PS, Matheus MG, Griesemer DA, et al. (2010) Natural history of Christianson syndrome. Am J Med Genet 152A(11): 2775-2783. 